\title{
CARACTERIZAÇÃO METODOLÓGICA DAS PESQUISAS SOBRE O ESTRESSE OCUPACIONAL E A SÍNDROME DE BURNOUT: UMA REVISÃO SISTEMÁTICA DE LITERATURA
}

\author{
METHODOLOGICAL CHARACTERIZATION OF RESEARCH ON \\ OCCUPATIONAL STRESS AND BURNOUT: A SYSTEMATIC \\ LITERATURE REVIEW
}

Renato Américo Dantas Camilo de Souza ${ }^{1}$ Jonathan Bento Cavalcanti ${ }^{2}$ Edwirde Luiz Silva Camêlo ${ }^{3}$

RESUMO: Este estudo buscou revisar sistematicamente a literatura acadêmica a fim de responder a seguinte questão norteadora: "Quais os parâmetros metodológicos adotados em pesquisas sobre o Estresse Ocupacional e a Síndrome de Burnout nos últimos 2 anos de produção científica internacional?". Incluíram-se artigos publicados entre janeiro de 2019 e janeiro de 2021 nas plataformas SciELO e LILACS/BVS. Dos 3314 trabalhos inicialmente encontrados, 150 foram selecionados após a aplicação das etapas de filtragem, sendo as maiores prevalências observadas: artigos de $2019(77 / 66,7 \%)$, de autores brasileiros $(100 / 66,7 \%)$, da área de Enfermagem (61/40,7\%), com desenho Transversal (111/74\%) e Quantitativo(138/92\%). Nesse sentido, reitera-se a importância de se conhecer as principais características e delineamentos do referencial bibliográfico do Estresse Ocupacional e da Síndrome de Burnout a fim de que se possa garantir a continuidade de pesquisas futuras sobre o tema.

Palavras chave: Burnout. Estresse ocupacional. Revisão de literatura. Saúde mental.

ABSTRACT: This study sought to systematically review the academic literature in order to answer the following guiding question: "What are the methodological parameters adopted in research on Occupational Stress and Burnout Syndrome in the last 2 years of international scientific production?". Articles published between

\footnotetext{
1 Graduando de Psicologia pela Universidade Estadual da Paraíba - UEPB, macrenato2010@gmail.com.

${ }_{2}^{2}$ Graduando de Psicologia pela Universidade Estadual da Paraíba - UEPB, jonathan.ifpb@gmail.com.

${ }^{3}$ Professor de Estatística pela Universidade Estadual da Paraíba - UEPB, edwirde@uepb.edu.br.
} 
January 2019 and January 2021 on the SciELO and LILACS / VHL platforms are included. Of the 3314 completed papers, 150 were obtained after applying the filtering steps, with the highest prevalence observed: articles from 2019 (77/ 66.7\%), by Brazilian authors (100 / 66.7\%), from the Nursing area (61 / 40.7\%), with Transversal (111/74\%) and Quantitative (138 / 92\%) design. In this sense, it is reiterated the importance of knowing the main characteristics and designs of the bibliographic reference of Occupational Stress and Burnout Syndrome to ensure the continuity of future research on the theme.

Keywords: Burnout. Occupational stress. Literature review. Mental health. 


\section{INTRODUÇÃO}

Apesar de ainda não constar no CID-10 (Classificação Estatística Internacional de Doenças e Problemas Relacionados com a Saúde) e no DSM-5 (Manual Diagnóstico eEstatístico de Transtornos Mentais), há toda uma bibliografia especializada que se debruça sobre a questão do estresse ocupacional, apreendido, em linhas gerais, na medida das tensões advindas de uma relação mal equilibrada entre determinado indivíduo e seu ambiente de trabalho, cujos fatores estressantes (de ordem organizacional, próprios da função exercida, ou de ordem individual, atrelados à dimensão psicofisiológica do trabalhador) tendem a minar o bem-estar e a saúde do trabalhador, trazendo para sua vida pessoal uma série de prejuízos emocionais, psicológicos e físicos (RIBEIRO et al., 2019).

A síndrome de Burnout é uma enfermidade multidimensional caracterizada pela presença de exaustão emocional, desumanização (despersonalização) e baixa realização pessoal no trabalho. $O$ estresse vivido no ambiente de trabalho acumulase ao ponto de cronificação dos sintomas e as estratégias de coping (adaptação) se esgotam, fazendo com que o profissional seja gradualmente menos eficiente no desempenho de sua função aomesmo tempo em que também atrapalha sua vida fora do trabalho (ALVARES et al., 2020).

As dinâmicas contemporâneas de trabalho e sociedade constantemente acumulam novas fontes de estresse. Conflitos de interesse entre empregado e empregador (BORGES, 2017), sobrecarga (ARAGÃO et al., 2021; ARAÚJO et al., 2020; SANTOS, LIMA, \& OLIVEIRA, 2019), dupla jornada (SOARES et al., 2021), natureza insalubre da profissão, conflitos e preconceitos étnico-raciais (KOTZE \& MASSYN, 2019) constituem alguns dos diversos atravessamentos que promovem o acúmulo de estresse. A presença de síndromes provenientes do excesso de estresse laboral como a de Burnout vem aumentando nos últimos anos, essa tendência provavelmente é fruto de trabalhos em ambientes de trabalho frios, 
competitivos, hostis e altamente exigentes (ALVARES et al., 2020) dentre outros elementos supracitados.

Profissões que requerem um nível de qualificação mais baixo e apresentam um alto índice de rotatividade podem apresentar um alto nível de estresse devido à precarização do trabalho (ROCHA \& ARAÚJO, 2016). Além disso, profissionais da saúde como médicos e enfermeiros que são submetidos a regimes de trabalho com uma carga horária intensa frequentemente noturna em contato constante com a dor e a morte, são um dos mais afetados pelo estresse ocupacional e consequentemente pela síndrome de Burnout (SANTOS, LIMA, \& OLIVEIRA, 2019). Outra área bastante afetada é a dos professores, sendo cobrados constantemente pela execução de uma série de multitarefas como dar aula, assumir cargos administrativos na escola, lidar com falta de interesse e indisciplina dos alunos, manter um canal de comunicação com os responsáveis pelos alunos, dentre outras responsabilidades que podem surgir de acordo com a situação. Todas estas responsabilidades se acumulam fazendo com o que o profissional se torne alvo de vários estressores psicossociais (SANTOS, LIMA, \& OLIVEIRA, 2019).

Nesse sentido, pretende-se no presente estudo aprofundar a compreensão acerca dos delineamentos metodológicos mais recorrentemente empregados pela bibliografia científica de referência sobre a Síndrome de Burnout e o Estresse Ocupacional. Para este fim, buscou- se revisar sistematicamente a literatura acadêmica presente nas principais bases de dados como intuito de responder a seguinte questão norteadora: "Quais os parâmetros metodológicos adotados em pesquisas sobre o Estresse Ocupacional e a Síndrome de Burnout nos últimos 2 anos de produção científica internacional?" Fornecendo uma catalogação extensiva e sistemática das principais escolhas metodológicas já empregadas pelos pesquisadores da área, tal proposta de investigação justifica-se em função da relevância social associada ao presente objeto de estudo, contribuindo para a elaboração de novas pesquisas sobre a Síndrome de Burnout e o Estresse Ocupacional. 


\section{METODOLOGIA}

A presente revisão sistemática incluiu artigos publicados entre janeiro de 2019 e fevereiro de 2021 nas bases de dados SciELO e LILACS/BVS, coletados por meio daaplicação dos seguintes descritores do DECS/MeSH: "Burnout" OR "Esgotamento Profissional" OR "Occupational Stress" OR "Estresse ocupacional". Após a realização da busca eletrônica dos materiais, procedeu-se à filtragem dos artigos através de três etapas de triagem sequenciais.

$\mathrm{Na}$ triagem inicial, incluíram-se: (1) artigos disponibilizados de forma integral, gratuita e com livre acesso; (2) publicações entre 01/01/2019 e 01/02/2021; (3) textos em Inglês e Português. Na segunda triagem, excluíram-se: (1) estudos de validação e de avaliação de testes psicométricos; (2) capítulos de livros e editoriais; (3) dissertações, teses demestrado e doutorado; (4) estudos teóricos e revisões de literatura. Por fim, na última etapa de triagem foram excluídas: (1) publicações em que o Estresse Ocupacional ou a Síndrome de Burnout não aparecem de forma explícita como parte do objeto do estudo; (2) publicações duplicadas entre as bases de dados.

\section{RESULTADOS E DISCUSSÃO}

A partir da busca eletrônica nos periódicos, coletou-se um montante de 3314 manuscritos (SciELO: 1215; LILACS: 2099), sendo 3164 excluídos após a aplicação das 3 etapas de triagem, assim compondo-se uma seleção final de 150 artigos (SciELO: 66; LILACS: 84) conforme se verifica na Figura 1 abaixo. 
Figura 1: Fluxograma das etapas de filtragem dos artigos selecionados na Revisão Sistemática.

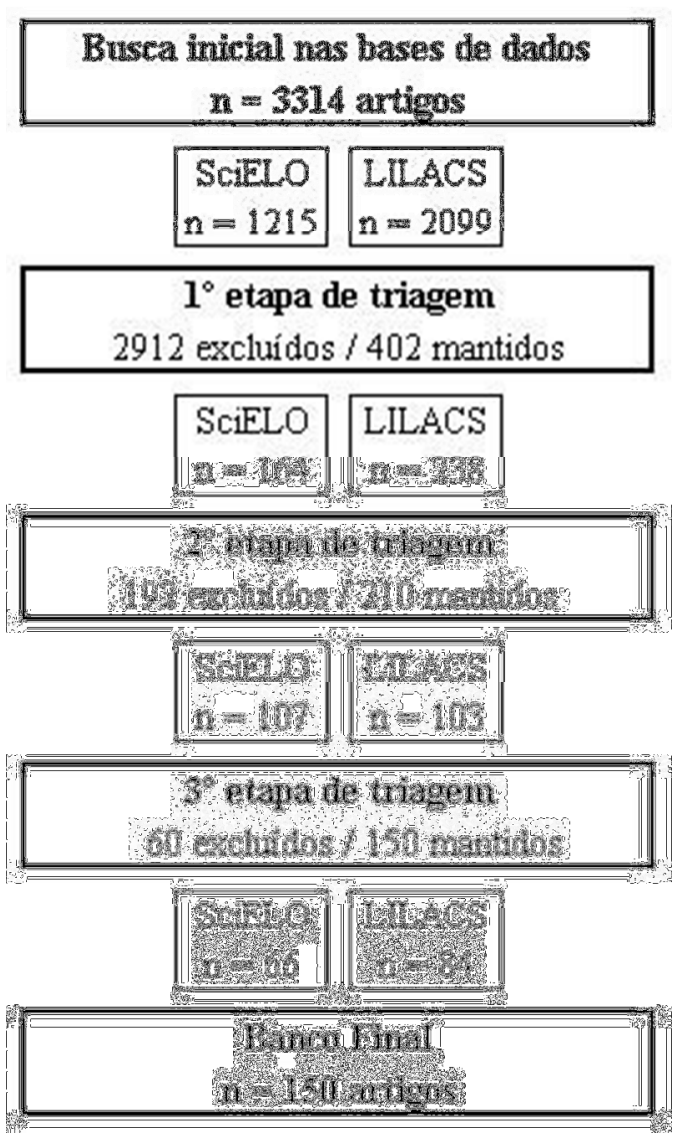

Fonte: Elaboração Própria.

As informações metodológicas presentes nos 150 artigos que compuseram a seleção final desta revisão sistemática foram coletadas e compiladas em uma tabela descritiva (ver Tabela 1), considerando-se os seguintes tópicos de interesse: Ano de publicação; País onde a pesquisa foi realizada; Área de estudo dos pesquisadores; Desenho do estudo e Metodologia de pesquisa. 
Tabela 1: Informações coletadas dos artigos selecionados da Revisão Sistemática.

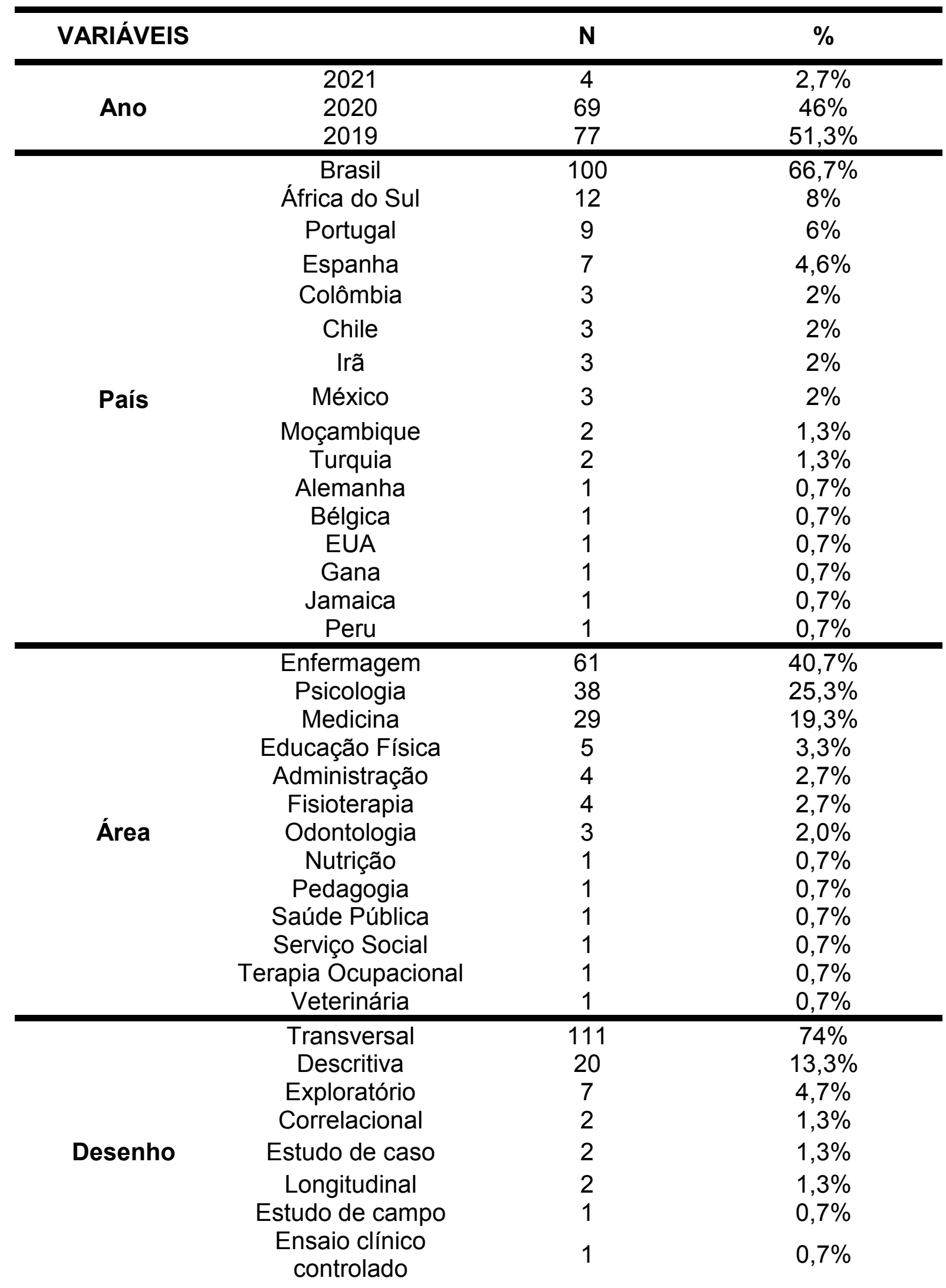




\begin{tabular}{cccc} 
& Ensaio clínico & 1 & $0,7 \%$ \\
& randomizado & 1 & $0,7 \%$ \\
& Fenomenológico & 1 & $0,7 \%$ \\
& Relato de & 1 & $0,7 \%$ \\
\hline & experiência & 1 & $92 \%$ \\
& Seccional & 138 & $4,7 \%$ \\
\multirow{2}{*}{ Metodologia } & Quantitativa & 7 & $3,3 \%$ \\
\hline TOTAL & Qualitativa & 5 & $\mathbf{1 0 0 \%}$ \\
\hline
\end{tabular}

Fonte: Elaboração Própria.

Considerando o recorte temporal previamente estabelecido nesta revisão sistemática para seleção dos manuscritos coletados nas bases de dados eletrônicas, observa-se umafrequência média de 6 artigos publicados ao mês entre 01/01/2019 e 01/02/2021. Tal evidência parece indicar um significativo interesse acadêmico em pesquisas recentes envolvendo a temática, corroborando o progressivo aumento das pesquisas sobre os transtornos biopsicossociais associados à presença de estressores no contexto laboral (MOREIRA, 2019). Este incremento na produção científica parece despontar como consequência do crescimento histórico dos casos de Burnout, que trazem níveis expressivos de incapacidade funcional e riscos à saúde do trabalhador (MORENO et al, 2011), assim sublinhando a importância de uma maior aproximação entre as produções da comunidade científica e as contingências e demandas da realidade sociocultural e político-econômica no qual esta se insere e dialoga.

Somadas, as publicações produzidas por pesquisadores nativos de países de língua portuguesa alcançam o montante de 111 artigos, 74\% do total considerado nesta revisão sistemática. De fato, não pode ser desconsiderado o fato de a língua portuguesa ter composto, juntamente com a língua inglesa, um dos critérios de seleção para triagem dos artigos que foram selecionados nesta revisão sistemática, o que poderia corroborar a elevada porcentagem de pesquisas atribuídas a pesquisadores do Brasil (100 / 66,7\%), Portugal (9 / 6\%) e Moçambique (2 / 1,3\%). Contudo, o argumento acima levantado parece não justificar a presença significativamente diminuta de artigos associados a autores nativos de países de língua inglesa. Nesse sentido, os dados levantados indicam um significativo 
protagonismo ocupado pela comunidade acadêmica brasileira no âmbito da produção de pesquisas e estudosenvolvendo o Estresse Ocupacional e a síndrome de Burnout.

No que concerne à proeminência de publicações ligadas à Enfermagem (61) $40,7 \%$ ), reitera-se a elevada proximidade com que os pesquisadores e profissionais lotados nesta área reiteradamente dialogam, do ponto de vista teórico e prático, com os diversos aspectosenvolvidos com a temática (VALERETTO \& ALVES, 2013), não apenas na condição de cuidadores de pessoas acometidas com Burnout como, sobretudo, enquanto vítimas cotidianas dos efeitos e comprometimentos dessa condição (FREITAS et al, 2014). Ademais, a presença massiva de pesquisas produzidas por autores de diversas áreas de saúde, em claro contraste com os 2,7\% pertinentes aos artigos de Administração, sublinha a necessidade de que sejam implementadas, no âmbito da gestão de saúde pública e privada, estratégias e açõesvoltadas à preservação da saúde mental, da ergonomia e da qualidade de vida dos profissionais da saúde quando inseridos em seus respectivos ambientes de trabalho.

Do ponto de vista metodológico, observa-se uma significativa prevalência de artigos com design Transversal (111 / 74\%) e Quantitativos (138 / 92\%). Tal preferência fortemente acentuada parece sugerir uma tendência a recortes mais mensuráveis no âmbito dos estudos da síndrome de Burnout, que por sua vez já é bem documentada na literatura com uma série de instrumentos validados disponíveis para a aferição do grau e incidência do transtorno (ARAGÃO, 2021; BATALHA et al. 2020; PÉREZ-FUENTE, 2019).Os aspectos que envolvem o estresse laboral e o desenvolvimento da síndrome de Burnout são multifatoriais, com métodos quantitativos bastante confiáveis para analisar cada um deles (ALVARES et al., 2020; ARAGÃO et al., 2021; ARAÚJO et al., 2020; BORGES, 2017; SANTOS, LIMA, \& OLIVEIRA, 2019). Embora este seja um indício de que os estudos sobre tais temáticas relacionadas à saúde do trabalhador tenham avançado bastante, a ausência de estudos mais exploratórios de cunho qualitativo e que acompanhem o objeto de estudo por períodos mais prolongados através de um desenho longitudinal pode indicar certa carência no modo como o assunto é abordado. 


\section{CONSIDERAÇÕES FINAIS}

O presente estudo buscou contribuir para a ampliação, sistematização e consolidação do conhecimento acerca das múltiplas características e delineamentos que permeiam a recente bibliografia científica, produzida em âmbito internacional, sobre os fenômenos do Estresse Ocupacional e da Síndrome de Burnout. Esperase, assim, fornecer aos interessados na temática um amplo panorama de escolhas metodológicas, facilitando os processos de tomada de decisão comumente envolvidos no decorrer da produção de pesquisas científicas.

Considerando o atual contexto sócio-histórico de significativo agravamento das condições políticoeconômicas de vida social experimentados no Brasil e no mundo emfunção da pandemia da COVID-19, reitera-se a urgente necessidade de que sejam priorizadas estratégias de enfrentamento assertivas e humanizadas frente ao fenômeno do Esgotamento Laboral, em especial para os profissionais de saúde. Conclui-se, portanto, que conhecer o histórico recente das práticas acadêmicas mais recorrentemente envolvidas na produção do referencial bibliográfico do Estresse Ocupacional e da Síndrome de Burnout reveste-se de fundamental importância para o fomento de futuras investigações relacionadas aos fenômenos supracitados, na medida em que se facilita o acesso de pesquisadores ao acervo das práticas metodológicas consolidadas pela comunidade científica. 


\section{REFERÊNCIAS BIBLIOGRÁFICAS}

ABACAR, M.; ALIANTE, G.; \& NAHIA, I. A. A. Fontes de estresse ocupacional e estratégias de enfrentamento em professores moçambicanos do ensino básico. Saúde e Pesquisa. v. 13, n. 1. Maringá/PR: 2021.

ACHKAR, A. M. N. et al. Life Satisfaction and Academic Performance of Elementary School Students. Psico-USF. v. 24, n. 2, p. 323-335. Bragança Paulista/SP: 2019.

ÁLVARES, M. E. M. et al. Síndrome de burnout entre profissionais de saúde nas unidades de terapia intensiva: um estudo transversal com base populacional. Rev. bras. ter. intensiva., v. 32, n. 2, p. 251-260. São Paulo: 2020.

ALVES, M. C. Cardozo. et al. Prevalência de esgotamento profissional em técnicos em enfermagem de uma unidade de Terapia Intensiva Adulto. Rev. Bras. Enferm. v. 74, supl. 3, e20190736. 2021.

ALIANTE, G.; \& ABACAR, M. Estresse ocupacional em formadores de professores do ensino básico: estudo com profissionais do Instituto de Formação de Professores Primários de Nampula- Moçambique. Pesquisas e Práticas Psicossociais. v. 15, n. 1. 2020.

ANDRADE, A. L.; \& OLIVEIRA, M. Z. Apego e o Efeito mediador da Autoeficácia e Satisfaçãocom Vida na Expectativa de Sucesso de Universitários. Psicología, Conocimiento y Sociedad. v. 10,n. 1, p. 34-53. Porto Alegre/RS: 2020.

ARAGÃO, N. S. C. et al. Síndrome de Burnout e Fatores Associados em Enfermeiros de Unidade de Terapia Intensiva. Rev. Bras. Enferm. v. 74, supl. 3, e20190535. Brasília: 2021.

ARAÚJO, A. F. et al. Occupational stress of nurses from the Mobile Emergency Care Service. Rev. Bras. Enferm. v. 73, supl. 1, e20180898. Brasília: 2020.

ASSUNÇÃO, H.; \& MARÔCO, J. Utilização de medicamentos em estudantes universitários com Burnout. Psic., Saúde \& Doenças [online]. v. 21, n. 1, p. 15-21. 2020.

AYISI-BOATENG, N. K. et al. A cross-sectional self-assessment of burnout amongst a sample of doctors in Ghana. African Journal of Primary Health Care \& Family Medicine. v. 12, n. 1, a2336.2020.

BABIC, A.; GILLIS, N.; \& HANSEZ, I. Work-to-family interface and well-being: The role of workload, emotional load, support and recognition from supervisors. SA Journal of Industrial Psychology. v. 46, n. 0, a1628. 2020.

BAKIOĞLU, F.; \& KIRAZ, Z. Burnout and Wellbeing of Teacher Candidates: The Mediator Role of Cynicism. Annals of psychology. v. 35, n. 3, p. 521-528. 2019.

BALDONEDO-MOSTEIRO, M. et al. Burnout syndrome in Brazilian and Spanish nursing workers. Rev. Latino-Am. Enfermagem. v. 27. e3192. 2019.

BARDAQUIM, V. A. et al. Stress and cortisol levels among members of the nursing team. Rev. Bras.Enferm. v. 73, supl.1, e20180953. Brasília: 2020.

BATALHA, E. et al. Satisfação por compaixão, burnout e estresse traumático secundário em enfermeiros da área hospitalar. Revista Portuguesa de Enfermagem de Saúde Mental. n. 24, p. 25- 33. 2020. 
BEZERRA, C. M. B. et al. Prevalência do estresse e síndrome de Burnout em enfermeiros no trabalhohospitalar em turnos. REME - Rev Min Enferm. v. 23. e-1232. 2019.

BICALHO, C. C. F. Prevalência do burnout em atletas de futebol da categoria sub-20 ao longo de uma temporada esportiva. J. Phys. Educ., v. 31, e3160. 2020.

*BORGES, A. Os novos horizontes de exploração do trabalho, de precariedade e de desproteção. Cadernos do CEAS: Revista crítica de humanidades, n. 239, p. 713-741, 2017.

BORGES, E. M. N. et al. Fadiga por compaixão em enfermeiros de urgência e emergência hospitalar de adultos. Rev. Latino-Am. Enfermagem. v. 27. e3175. 2019.

BRANCO, F. M. F. C. et al. Síndrome de Burnout entre trabalhadores de uma universidade na fronteira franco-brasileira. Rev. Fun. Care Online. n.12, p. 393-399. 2020.

BUCETA, M. I.; BERMEJO, J. C.; \& VILLACIEROS, M. Enhancer elements of compassion satisfaction in healthcare professionals. Annals of psychology. v. 35, n. 2, p. 323-331. 2019.

CALCIDES, D. A. P. et al. Burnout Syndrome in medical internship students and its prevention withBalint Group. Rev. Assoc. Med. Bras. v. 65, n. 11, p. 1362-1367. 2019.

CAMPOS, F. M. et al. Estresse ocupacional e saúde mental no trabalho em saúde: desigualdades degênero e raça. Cad. Saúde Colet., v. 28, n. 4, p. 579-589. 2020.

CARLOTTO, M. S.; \& CÂMARA, S. G. Burnout Syndrome in Public Servants: Prevalence and association with Occupational Stressors. Psico-USF. v. 24, n. 3, p. 425-435. 2019.

CARVALHO, A. E. L. et al. Stress of nursing professionals working in pre-hospital care. Rev. Bras. Enferm. v. 73, n. 2, e20180660. Brasília: 2020.

CARVALHO, D. P. et al. Workloads and Burnout of nursing workers. Rev. Bras. Enferm. v. 72, n.6, p. 1435-1441. Brasília: 2019.

CARVALHO, L. F. et al. Pathological personality as predictor of work engagement, job satisfaction, and burnout in a community sample. Revista Psicologia: Organizações e Trabalho. v. 20, n. 1, p. 877-882. 2020.

CASTRO, C. S. A. A. et al. Síndrome de burnout e engajamento em profissionais de saúde: um estudotransversal. Rev. bras. ter. intensiva [online]. v. 32, n.3, p.381-390. São Paulo: 2020.

CASTRO, J. R. et al. Estresse ocupacional e engajamento em profissionais de saúde Bucal. Rev. Bras. Promoç. Saúde. v. 32, n. 9. 2019.

CAVALCANTE, J. L. et al. Estresse ocupacional dos funcionários de uma universidade pública. Enferm. Foco. v. 10, n. 4, p. 108-115. 2019.

CAZOLARI, P. G. et al. Níveis de Burnout e Bem-Estar de Estudantes de Medicina: um Estudo Transversal. Rev. bras. educ. med. v. 44, n. 4. e125. Brasília: 2020.

CORDEIRO, R. et al. Stress e estratégias de coping em profissionais de saúde. Revista Portuguesa de Enfermagem de Saúde Mental. n. 7, p. 09-16. 2020.

CORDIOLI, D. F. C. et al. Occupational stress and work engagement in primary health care workers. Rev. Bras. Enferm. v. 72, n. 6, p. 1580-7. Brasília: 2019.

CORREAA, J. S. et al. Bem-estar no trabalho e síndrome de Burnout: Faces opostas no labor penitenciário. Revista de Administração Mackenzie. v. 20, n. 3. 2019. 
COSTA, M. V. C. et al. Exercícios de alongamento na percepção de estresse em profissionais de enfermagem: estudo clínico randomizado. Cad. Bras. Ter. Ocup. v. 27, n. 2, p. 357-366. São Carlos/SP: 2019.

COSTA, V. H. L. B.; BORSA, J. C.; \& DAMÁSIO , B. F. Relações entre Burnout, Traços dePersonalidade e Variáveis Sociodemográficas em Trabalhadores Brasileiros. Psico-USF., v. 25, n. 3, p. 439-450. Bragança Paulista/SP: 2020.

CRUZ, G. F. et al. Estresse ocupacional e fatores associados: um estudo em professores. Saúde ePesquisa. v. 13, n. 3, p. 583-592. Maringá/PR: 2020.

CRUZ, S. P. et al. Fatores relacionados à probabilidade de sofrer problemas de saúde mental emprofissionais de emergência. Rev. Latino-Am. Enfermagem. v. 27. e3144. 2019.

DALMOLIN, G. L. et al. Prazer e sofrimento em trabalhadores da atenção primária à saúde do Brasil. Rev Cuid. v. 11, n. 1. e851. 2020.

DE VINE, J.; \& MORGAN, B. The relationship between personality facets and burnout. SA Journalof Industrial Psychology. v. 46, n. 0. a1786. 2020.

DORNELES, A. J. A. et al. Sociodemographic and occupational aspects associated with burnout inmilitary nursing workers. Rev. Bras. Enferm. v. 73, n. 2. e20180350. Brasília: 2020.

DORNELLES, T. M. et al. Qualidade de vida profissional e coping num hospital de referência paravítimas de violência sexual. Texto Contexto Enferm. v. 29. e20190153. 2020.

DUTRA, H. S. et al. Burnout entre profissionais de enfermagem em hospitais no Brasil. Rev. Cuid. v.10, n. 1. e585. 2019.

FARIA, S. et al. Saúde mental dos enfermeiros: Contributos do Burnout e engagement no trabalho. Revista Portuguesa de Enfermagem de Saúde Mental. n. 22, p. 09-18. 2019.

FERNANDES, I. M. C. et al. Níveis de ansiedade, depressão e estresse em funcionários de uma instituição de ensino superior pública do interior do estado de São Paulo. Rev. Bras. Med. Trab. v. 17, n. 4, p. 530-536. 2019.

FERREIRA, M. S. G; \& ANDERSON, M. I. P. Sobrecarga de trabalho e estresse: relato sobre um grupo de apoio à saúde do trabalhador em uma Unidade de Saúde da Família. Rev. Bras. Med. Fam. Comunidade. v.15, n. 42. e2188. Rio de Janeiro: 2020.

FIGUEIROA, G. B. et al. Síndrome de Burnout entre profissionais de um serviço de atendimento móvel de urgência do Paraná. Cogitare enferm. v. 24. e61917. 2019.

FILHO, I. M. M. et al. Associação de estresse ocupacional e uso de psicotrópicos por docentes da áreada saúde. Revista Brasileira em Promoção da Saúde. v. 32. e9007. 2019.

FODOR, D. P. et al. Intensity matters: The role of physical activity in the Job DemandsResources model. Journal of Work and Organizational Psychology. v. 36, n.3, p. 223-229. Madrid: 2020.

FONSECA, S.; QUEIRÓS, C.; \& MARTINS, V. Saúde ocupacional na ferrovia: trabalho por turnos,Burnout, sono e interação trabalho-família. Psicologia, Saúde \& Doenças. v. 21, n. 1. 2020.

FONTENELE, R. M. et al. Fatores de Vulnerabilidade para o Sofrimento Psíquico Relacionado ao Trabalho de Agentes Administrativos. Revista Brasileira de Ciências da Saúde. v. 23, n. 2, p. 115-122. 2019. 
${ }^{*}$ FREITAS, A. R. Impacto de um programa de atividade física sobre a ansiedade, depressão, estresse ocupacional e síndrome de Burnout dos profissionais de enfermagem no trabalho. Rev. Latino-Am. Enfermagem. v. 22, n. 2, p. 332-336. 2014.

GALDINO, M. J. Q. et al. Burnout among nursing students: a mixed method study. Invest. Educ. Enferm. v. 38, n.1. e07. 2020.

GOEBEK, D. K.; \& CARLOTTO, M. S. Preditores sociodemográficos, laborais e psicossociais da Síndrome de Burnout em docentes de educação a distância. Avances en Psicologia Latinoamericana. v. 37, n. 2, p.295-311. 2019.

GUISSI, P. C. et al. Os fatores psicossociais no trabalho e estresse entre os profissionais de enfermagem de uma Central de Materiais Esterilizados. Rev Bras Med Trab. v. 17, n. 4. 2019.

GUÍZAR-SÁNCHEZ, D. et al. Self-perceived health in psychiatrists and psychiatry trainees: the roleof perfectionism and distress. Salud Mental. v. 43, n. 5, p. 201-208. 2020.

GULER, Y. et al. Burnout syndrome should be not be underestimated. Rev Assoc Med Bras. v. 65, n.11, p. 1356-1360. 2019.

GUTIÉRREZ, K. P. M. et al. Burnout syndrome in nursing professionals in Punta Arenas, Chile. Texto Contexto Enferm. v. 29. e20190273. 2020.

HOSEINABADI, T. S. et al. Burnout and its influencing factors between frontline nurses and nurses from other wards during the outbreak of Coronavirus Disease (COVID-19) in Iran. Invest. Educ. Enferm. v. 38, n. 2. e03. 2020.

HU, X.; SANTUZZI, A. M. \& BARBER, L. K. Disconnecting to Deatch: The Role of Impaired Recovery in Negative Consequences of Workplace Telepressure. Journal of Work and Organizational Psychology. v. 35, n.1, p. 9-15. 2019.

HUTCHINSON, T. A. et al. Stress, Burnout and Coping among Emergency Physicians at a Major Hospital in Kingston, Jamaica. West Indian Med. J. v. 63, n. 3, p. 262-266. 2020.

JÚNIOR, E. P. B. et al. Predisposition to Burnout Syndrome in prison Officers. O Mundo da Saúde. v. 42, n. 3, p. 530-541. São Paulo: 2019.

KHESWA, J. Factors and effects of work-related stress and burnout on the well-being of social workers in the Eastern Cape province South Africa. S A Journal of Industrial Psychology. v. 45, n. 0. a1661. 2019.

KOTZE, M.; \& MASSYN, L. The influence of employees' cross-cultural psychological capital on workplace psychological well-being. S A Journal of Industrial Psychology. v. 45, n. 0. a1660. 2019. LEITE, T. I. A. et al. Prevalência e fatores associados da síndrome de Burnout em docentes universitários. Rev Bras Med Trab. v., 17, n. 2, p. 170-179. 2019.

LOIOLA, E, \& MARTINS, M. C. Autoeficácia do trabalho e síndrome de Burnout em profissionais de Enfermagem. Psicologia, Saúde \& Doenças. v. 20, n.3, p. 813-823. 2019.

LOPES, H. L., \& BARBOSA, S. C. Qualidade de Vida Profissional: o que mantém o bem-estar psíquico de bombeiros? Revista Psicologia: Organizações e Trabalho. v. 20, n. 2. 2020.

LOPES, A. R.; \& NIHEI, O. K. Burnout among nursing students: predictors and association with empathy and self-efficacy. Rev. Bras. Enferm. v. 73, n. 1. e20180280. Brasília: 2020.

LOURENÇO, V. P. et al. Relação entre presenteísmo, síndrome de Burnout e liderança ética em organizações escolares. Fractal: Revista de Psicologia. v. 32, n. esp., p. 218-226. 2020. 
MAGRI, L.V. et al. Temporomandibular disorders and Burnout Syndrome in students of the eight period of dentistry course. RVO, Rev Gaúch Odontol. v. 67. e20190055. 2019.

MAILA, S.; MARTIN, P. D.; \& CHIPPS, J. Professional quality of life amongst nurses in psychiatric observation units. S. Afr. J. Psychiat. v. 26, n. 0. a1553. 2020.

MARSHALL, G. H.; \& STEPHENSON, S. M. Burnout and turnover intention among electronicsmanufacturing employees in South Africa. SA Journal of Industrial Psychology. v. 46, n. 0. 2020.

MOHEBBI, Z. et al. The effect of aerobic Exercise on Occupational stress of female nurses: Acontrolled Clinical trial. Invest. Educ. Enferm. v. 37, n. 2, e05. 2019.

MENEGATTI, M, S. Et al. Estresse e estratégias de coping utilizadas por residentes de Enfermagem. REME - Rev. Min. Enferm. v. 24. e-13292020. 2020.

MONTERROSA-CASTRO, A. et al. Estrés laboral, ansiedad y miedo al COVID-19 en médicos generales colombianos. MedUNAB. v. 23, n. 2, p. 214-232. 2020.

*MOREIRA, T. S. V. O impacto do estresse ocupacional e Síndrome de Burnout entre militares do Exército Brasileiro. EsSEX: Revista Científica. v. 2, n. 3, p. 29-35. 2019.

MOREIRA, A. S.; \& LUCCA, S. R. Psychosocial factors and Burnout Syndrome among mental health professionals. Rev. Latino-Am. Enfermagem. v. 28. e3336. 2020.

*MORENO, F. N. et al. Estratégias e intervenções no enfrentamento da síndrome de Burnout. Rev. enferm. UERJ. v. 19, n. 1, p. 140-5. Rio de Janeiro: 2011.

MOURA, E. C.; FURTADO, L.; \& SOBRAL, F. Epidemia de Burnout durante a pandemia de covid- 19: o papel da LMX na redução do burnout dos médicos. RAE - Revista de Administração de Empresas. v. 60, n. 6, p. 426-436. São Paulo: 2020.

MUNHOZ, O. L. et al. Estresse ocupacional e burnout em profissionais de saúde de unidades de perioperatório. Acta Paul. Enferm. v. 33, p. 1-7. e20190261. 2020.

NASCIMENTO, J. O. V. et al. Trabalho em turnos de profissionais de enfermagem e a pressão arterial, burnout e transtornos mentais comuns. Rev. Esc. Enferm, USP, v. 53, e03443. 2019.

NEUMANN, G. C.; \& CARLOTTO, M. S. Fatores Associados à Síndrome de Burnout em Motoristas de Transporte Coletivo de Passageiros. Revista Psicologia: Organizações e Trabalho. v. 20, n. 3, p. 1089-1096. 2020.

NGIRANDE, H.; \& MJOLI, T. Q. Uncertainty as a moderator of the relationship between job satisfaction and occupational stress. SA Journal of Industrial Psychology. v. 46, n. 0. 2020.

NOBRE, D. F. R. et al. Avaliação do burnout em enfermeiros de uma serviço de urgência geral. Rev. Bras. Enferm. v. 72, n. 8. p. 1533-1539. 2019.

NOVAES NETO, E. M.; XAVIER, A. S. G.; \& ARAÚJO, T. M. Factors associated with occupationalstress among nursing professionals in health services of medium complexity. Rev. Bras. Enferm. v. 73, n. 1. e20180913. Brasília: 2020.

OLIVEIRA, R. F. et al. Fatores associados à ocorrência da síndrome de Burnout entre estudantes de residências multiprofissionais. Revista Brasileira de Educação Médica. v. 44, n. 2. 2020.

OLIVEIRA, S. M. D.; HASSE, M.; \& TEIXEIRA, F. B. Fluxo do esgotamento: interrogando o 
processo de produção do tempo/cansaço no internato médico. Rev. bras. educ. med. v. $45, \mathrm{n}$. 1, e009.Brasília: 2021.

ORTIZ-FUNE, C.; KANTER, J. W.; \& ARIAS, M. F. Burnout in mental health professionals: theroles of psychological flexibility, awareness, courage, and love. Clínica y Salud. v. 31, n. 2. 2020.

PALENZUELA, P; DELGADO, N. \& RODRÍGUEZ, J. A. Exploring the relationship between contextual performance and burnout in healthcare professionals. Journal of work and organizational Psychology. v. 35, v. 2, p.115-121. 2019.

PASTURA, P. S. V. C. et al. Do Burnout à Estratégia de Grupo na Perspectiva Balint: Experiência com Residentes de Pediatria de um Hospital Terciário. Revista Brasileira de Educação Médica. v. 43, n.2, p. 32-39. 2019.

PAYNE, A. et al. Burnout and job satisfaction of nursing staff in a South African acute mental health setting. S. Afr. J. Psychiat. v. 26, n. 0. a1454. 2020.

PÉREZ-FUENTES, M. C. et al. Analysis of Burnout Predictors n Nursing: Risk and Protective Psychological Factors. The European Journal of Psychology Applied to legal Context. v. 11, n.1,p. 33-40. 2019.

PINHEIRO, J. P. et al. Associação da empatia e do estresse ocupacional com o Burnout em profissionais da atenção primária à saúde. Ciência \& Saúde Coletiva. v. 25, n. 9. 2020.

PIRES, D. A. et al. Dimensões de Burnout, Estratégias de Coping e Tempo de Prática como Atleta Federado em Jogadores Profissionais de Futebol. Cuadernos de Psicología del Deporte. v. 19, n. 2,p. 175-185. Murcia: 2019.

PORCIUNCULA, A. M. et al. Síndrome de Burnout em gerentes da Estratégia de Saúde da Família.Ciência \& Saúde Coletiva. v. 25, n. 4, p.1555-1565. 2020.

PRADO, M. F. S. N. et al. Avaliação da Síndrome de Burnout entre os estudantes do último ano do curso de medicina no Brasil. Arch. Health. Sci. v. 21, n.1, p. 41-46. 2019. RAMOS, F. R. S. et al. Intensity and frequency of moral distress in Brazilian nurses. Rev. Esc.Enferm. USP. v. 54. e03578. São Paulo: 2020.

REIS, C. D. Prevalência de síndroma de Burnout em médicos de família da Secção Regional Norte daOrdem dos Médicos. Rev Port Med Geral Farm. v. 35, n., p.176-84. 2019.

REIS, C. D. et al. Situações estressoras e estratégias de enfrentamento adotadas por enfermeiraslíderes. Acta Paul. Enferm. v. 33, p. 1-7. 2020.

*RIBEIRO, R. P. et al. Estresse ocupacional entre trabalhadores de saúde de um hospital universitário. Revista Gaúcha de Enfermagem, v. 39, 2018.

RIVAZ, M.; ASADI, F.; \& MANSOURI, P. Assessment of the Relationship between Nurses' Perception of Ethical Climate and Job Burnout in Intensive Care Units. Invest. Educ. Enferm. v. 38, n. 3. e12. 2020.

ROCHA, L. J. et al. Esgotamento profissional e satisfação no trabalho em trabalhadores do setor de emergência e terapia intensiva em hospital público. Rev Bras Med Trab. v. 17, n. 3, p. 300-12. 2019. ROCHA, R. P. S. et al. Características do trabalho e estresse ocupacional entre enfermeiros hospitalares. Enferm. Foco. v. 10, n. 5, p. 51-57. 2019.

${ }^{*}$ ROCHA, C. J.; ARAÚJO, G. F. Percepção do estresse em operadoras de telemarketing. Psicol. Rev.,São Paulo, v. 10, n 32, nov. 2016. 
RODRIGUES, C. S. et al. Avaliação da prevalência da síndrome de Burnout em estudantes de medicina. Revista Brasileira de Educação Médica. v. 44, n. 4. e176. 2020.

ROMERO, M. P. Working conditions and the meaning of the academic profession in colombian university professors. Acta Colombiana de Psicología. v.22, n.2, p. 280-291. 2019.

ROSAS-NAVARRO, J. M. et al. Association between Burnout Syndrome and medical training by specialty in firstyear residents. Salud Mental. v. 43, n. 5, p. 227-233. 2020.

ROSSATO, G. et al. Estresse e resiliência no trabalho em servidores públicos federais. Enferm.Foco. v. 11, n. 2, p. 78-86. 2020.

SAAVEDRA, J.; MURVARTIAN, L.; \& VALLECILLO, N. Health and burnout of home health care assistants: Impact of a training intervention. Annals of psychology. v. 36, n 1, p. 30-38. 2020.

SACADURA-LEITE, E. et al. Condições de trabalho exaustão emocional elevada em enfermeiros no ambiente hospitalar. Rev Bras Med Trab. v.17, n.1, p.69-75. 2019.

SAIKAI, G. M. P. N.; NARDI, S. M. T.; \& SAIKAI, W. et al. Exaustão Emocional e Física deCuidadores Familiares. Rev. Fun. Care Online. v. 12, p. 1296-1302. 2020.

SANTANA, R. S. et al. Estresse ocupacional dos enfermeiros de urgência e emergência de umhospital público de Teresina (PI). Rev Bras Med Trab. v. 17, n.1, p.76-82. 2019.

SANTANA, L. C. et al. Aspecto psicossocial do ambiente de trabalho de profissionais de enfermagem segundo o modelo demanda-controle. Rev. Enferm. UERJ. v. 28. e50740. Rio de Janeiro: 2020.

SANTANA, L. C.; FERREIRA, L. A.; \& SANTANA, L. P. M. Occupational stress in nursing professionals of a university hospital. Rev. Bras. Enferm. v. 73, n. 2. e20180997. Brasília: 2020.

SANTOS, A. et al. Síndrome de Burnout e estilo de vida em estudantes de ensino médio. RevistaPortuguesa de Enfermagem de Saúde Mental. n. 21. 2019.

SANTOS, A. C. A. et al. Differences in perception of burnout syndrome among young athletes fromindividual and team sports. Motricidade. v. 16, n. 1, p. 39-46. 2020.

SANTOS, E. N. et al. Saúde do trabalhador no ambiente hospitalar: fatores de risco para síndrome deburnout. Revista Nursing. v. 22, n. 248, p. 25009-2513. 2019.

SANTOS, J. L. G. et al. Síndrome de Burnout entre enfermeiros de um Hospital Universitário. Revbaiana de enferm. v. 33. e29057. 2019.

SANTOS, K. M. R. et al. Depressão e ansiedade em profissionais de enfermagem durante a pandemiada covid-19. Esc. Anna Nery. v. 25, e20200370. Rio de Janeiro: 2021.

*SANTOS, J. D.; LIMA, M. E. S. \& OLIVEIRA, F. K. F. Síndrome de Burnout perante as categorias profissionais mais diagnosticadas: uma revisão integrativa. IV SIMPÓSIO INTERDISCIPLINAR EM SAÚDE E AMBIENTE, p. 46. 2019.

SERRA, M. V. et al. Musculoskeletal disorders, stress perception and physical activity in police officers. Fisioter. Pesqui. v. 27, n. 1, p. 22-27. São Paulo: 2020.

SILLERO-SILLERO, A.; \& ZABALEGUI, A. Analysis of the work environment and intention of perioperative nurses to quit work. Rev. Latino-Am. Enfermagem. v. 28. e3256. 2020.

SILVA, A. A. et al. Predictor variables for burnout among nursing professionals. Revista de 
psicologia. v. 37, n.1, p. 319-348. 2019.

SILVA, P. N. et al. Autopercepção do estresse ocupacional na equipe de enfermagem de um serviçode emergência. Journal Health NPEPS. v. 4, n. 2, p. 357-369. 2019.

SILVA, R. A. D. A. et al. Análise da satisfação de profissional e síndrome de Burnout em profissionais de saúde bucal no Sistema Único de Saúde de Sobral, Ceará. Rev Bras Med Trab. v. 17, n. 3, p. 313-24. 2019.

SILVA, A. P. F.; CARNEIRO, L. V.; \& RAMALHO, J. P. G. Incidência da síndrome de Burnout em profissionais de enfermagem atuantes em unidade de terapia intensiva. Rev. Pesq. Cuid. Fundam. v. 12, p. 915-920. 2020.

SILVA, E.; DIAS, P.; \& RODRIGUES, A. Satisfação laboral e burnout em assistentes gerontológicos. Psicologia, saúde \& doenças. v. 20, n. 3, p. 788-802.2019.

SILVA-JUNIOR, R. F. et al. Personalidade hardiness e fatores associados em profissionais da saúde atuantes em serviços que atendem pacientes críticos. Ciência \& Saúde Coletiva. v. 25, n. 1, p. 199- 209. 2020.

SILVEIRA, L. et al. Síndrome de Burnout em preceptores e residentes vinculados a programas de residência em saúde da família. Cogitare enferm. v. 25. e67248. 2020.

SOARES, D. S. et al. Influence of physical activity on military police officers burnout. J. Phys.Educ. v. 30, e3059, 2019.

*SOARES, S. S. S. et al. Dupla jornada de trabalho na enfermagem: dificuldades enfrentadas no mercado de trabalho e cotidiano laboral. Escola Anna Nery, v. 25, n. 3, 2021.

SOUSA, A. K. A. et al. Burnout syndrome and perceptions about safety climate among intensive care professionals. Rev. Rene. v. 21. e43868. 2020.

SOUSA, C. C. et al. Occupational stress and job dissatisfaction with health work. Psicologia: Reflexão e crítica. v.32, n.18. 2019.

SOUZA E SILVA, D. et al. Síndrome de Burnout em residentes multiprofissionais em saúde. Rev enferm UERJ. v. 27. e 43737. 2019.

SOUZA, C. G. V. M. et al. Qualidade de vida profissional na saúde: um estudo em Unidades de Terapia Intensiva. Estudos de Psicologia. v. 24, n. 3, p. 269-280. 2019.

SOUZA, M. B. C. A.; HELAL, D. H.; \& PAIVA, K. C. M. Análise descritiva das dimensões do burnout: um estudo com jovens trabalhadores. Cad. Bras. Ter. Ocup. v. 27, n.4. 2019.

SUÁREZ-COLORADO, $Y$. et al. The academic burnout, engagement, and mental health changesduring a school semester. Duazary. v. 16, n.1, p. 23-27. 2019.

SUPERVIA, P. U. \& BORDÁS, C. S. Relationship between intrinsic orientations and academicburnout in students. Psicologia Escolar e Educacional. v. 23. e188061. 2019.

TEIXEIRA, G. S. et al. Qualidade de vida no trabalho e estresse ocupacional da enfermagem em unidade de pronto atendimento. Texto \& Contexto Enfermagem. v. 28. e20180298. 2019.

TEIXEIRA, T. S. C.; MARQUEZE, E. C.; \& MORENO, C. R. C. Produtivismo acadêmico: quando ademanda supera o tempo de trabalho. Rev. Saúde Pública. v. 54, n. 117. 2020.

TELES, H. M. M. et al. Níveis de estresse e engagement laboral dos Assistentes Sociais em Portugal. Estudos de Psicologia. v. 24, n. 3, p. 258-268. 2019. 
TOMAZ, H. C. et al. Síndrome de Burnout e fatores associados em profissionais da Estratégia Saúdeda Família. Interface (Botucatu). v. 24, supl. 1. e190634. São Paulo: 2020.

TORRES, J. et al. Qualidade de vida profissional e fatores associados em profissionais de saúde. Psicologia, saúde \& doenças. v. 20, n.3, p. 670-681. 2019.

TUNUNU, A. F.; \& MARTIN, P. Prevalence of Burnout among nurses working at a psychiatrichospital in the Western Cape. Curationis. v. 43, n. 1. a2117. 2020.

ULGUIM, F. O. et al. Trabalhadores da saúde: risco cardiovascular e estresse ocupacional. Rev BrasMed Trab. v.17, n.1, p. 61-8. 2019.

*VALERETTO, F. A.; \& ALVES, D. F. Fatores desencadeantes do estresse ocupacional e da síndrome de Burnout em enfermeiros. Rev saúde física \& mental - UNIABEU. v. 3, n. 2. 2013.

VAN DER MERWE, L. J.; BOTHA, A.; \& JOUBERT, G. Burnout and associated factors in undergraduate medical students at a South African University. Afr. J. Health Professions. Educ. v. 12, n. 2, p. 62-67. 2020.

VAN DER MERWE, L. J.; BOTHA, A.; \& JOUBERT, G. Resilience and coping strategies of undergraduate medical students at the University of the Free State. S. Afr. J. Psychiat. v. 26, n. 0. a1471. 2020.

VASCONCELOS, E. M. et al. Predictive factors of Burnout syndrome in nursing students at a public university. Rev. Esc. Enferm. USP. v. 54. e03564. São Paulo: 2020.

VAZQUEZ, A. C. S.; SANTOS, A. S. S.; \& COSTA, P. V. C. Trabalho e Bem- Estar: Evidências da Relação entre Burnout e Satisfação de Vida. Avaliação psicológica. v. 18, n. 4. 2019.

VICHEZ-CORNEJO, J. et al. Burnout Syndrome in physicians of a hospital in the peruvian amazon. Rev. Fac. Med. Hum. v.19, n.4, p. 60-67. 2019.

VIDAL DE LA FUENTE, S. et al. Evaluation of mental health and occupational stress in Mexican medical residents. Salud Mental. v. 43, n. 5, p. 209-218. 2019.

WAGNER, L \& PATHER, M. K. Exploring resilience in family physicians working in primary health care in the Cape Metropole. African Journal of Primary Health Care \& Family Medicine. v.11, n. 1, a1982. 2019.

WEINBORN, R. M. et al. Burnout syndrome prevalence in veterinarians working in Chile. Austral J vet Sci. v. 21, p. 91-99. 2019.

ZANATTA, A. B. Estresse e enfrentamento de trabalhadores de centro de atenção psicossocial em uma cidade do interior do Estado de São Paulo. Rev Bras Med. v.17, n.1, p. 83 - 89. 2019.

ZANIN, C. E; ANGONESE, A. S. Identificação da síndrome de burnout em motoristas do transporte da saúde. Estudos Interdisciplinares de Psicologia. v. 10, n. 3, p. 26-42. 2019.

ZEIJLEMAKER, M.D. \& MOOSA, S. The prevalence of burnout among registrars in the Schools of Clinical Medicine at the University of the Witwatersrand, Johannesburg, South Africa. S Afr Med J. v. 109, n. 9, p.668-672. 2019.

ZÉTOLA, V. F. et al. Burnout syndrome: are stroke neurologists at a higher risk? Arq Neuropsiquiatr. v. 77, n. 2, p. 84-90. 2019. 